Bull. Korean Math. Soc. 46 (2009), No. 3, pp. 567-576

DOI 10.4134/BKMS.2009.46.3.567

\title{
HELICOIDAL SURFACES AND THEIR GAUSS MAP IN MINKOWSKI 3-SPACE II
}

\author{
Miekyung Choi, Young Ho Kim*, And Gi-Chan Park
}

\begin{abstract}
We classify and characterize the rational helicoidal surfaces in a three-dimensional Minkowski space satisfying pointwise 1-type like problem on the Gauss map.
\end{abstract}

\section{Introduction}

Nash's imbedding theorem enables us to view every Riemannian manifold as a submanifold of a Euclidean space. In that sense, one way to study a Riemannian manifold is to apply the theory of submanifolds in a Euclidean space. Since B.-Y. Chen ([3]) introduced the notion of finite type immersion of submanifolds in a Euclidean space late 1970's, many works have been carried out in this area. Further, the notion of finite type can be extended to any smooth functions on a submanifold of a Euclidean space or a pseudo-Euclidean space. In dealing with submanifolds of a Euclidean or a pseudo-Euclidean space, the Gauss map is a useful tool to examine the character of submanifolds in a Euclidean space. For the last few years, two of the present authors and D. W. Yoon introduced and studied the notion of pointwise 1-type Gauss map in a Euclidean or a pseudo-Euclidean space ([4], [5], [7], [8]), namely the Gauss map $G$ on a submanifold $M$ of a Euclidean space or a pseudo-Euclidean space is said to be of pointwise 1-type if

$$
\Delta G=F(G+C)
$$

for a non-zero smooth function $F$ on $M$ and a constant vector $C$, where $\Delta$ denotes the Laplace operator defined on $M$.

On the other hand, a helicoidal surface is well known as a kind of generalization of some ruled surfaces and surfaces of revolution in a Euclidean space or a Minkowski space ([1], [2], [6]). Recently, two of the authors, H. Liu and D. W. Yoon have classified the helicoidal surfaces with pointwise 1-type Gauss

Received July 11, 2008.

2000 Mathematics Subject Classification. Primary 53B25, Secondary 53C40.

Key words and phrases. helicoidal surfaces, harmonic Gauss map, Minkowski space.

* Supported by KOSEF R01-2007-000-20014-0 (2008). 
map in a Minkowski 3-space $\mathbb{L}^{3}([5])$. Then, we may have a natural question as follows:

What helicoidal surfaces have the harmonic Gauss map, that is, $\Delta G=0$ ? Or, what helicoidal surfaces satisfy equation (1.1) whether the function $F$ is non-zero or zero?

In this paper, we mainly focus on the study of the helicoidal surfaces with harmonic Gauss map in a Minkowski 3-space and find the all solution spaces of the so-called rational helicoidal surfaces satisfying (1.1). As a consequence, we have the following characterizations:

Theorem A. Let $M$ be a helicoidal surface with space-like or time-like axis in a Minkowski 3-space $\mathbb{L}^{3}$. Then, a plane is the only rational helicoidal surface with harmonic Gauss map.

Theorem B. There exists no rational helicoidal surface with harmonic Gauss map which has null axis in Minkowski 3-space $\mathbb{L}^{3}$.

Theorem C. Let $M$ be a rational helicoidal surface with time-like axis in a Minkowski 3-space $\mathbb{L}^{3}$. Then, the Gauss map $G$ of $M$ satisfies the condition $\Delta G=F(G+C)$ for some smooth function $F$ and constant vector $C$ if and only if $M$ is an open part of a plane, a circular cylinder, a right cone, a right helicoid of type II or a helicoidal surface of elliptic type in $\mathbb{L}^{3}$.

\section{Preliminaries}

Let $\mathbb{L}^{3}$ be a Minkowski 3-space with the Lorentz metric

$$
\langle\cdot, \cdot\rangle=-d x_{0}^{2}+d x_{1}^{2}+d x_{2}^{2},
$$

where $\left(x_{0}, x_{1}, x_{2}\right)$ is a system of the canonical coordinates in $\mathbb{R}^{3}$. Let $M$ be a connected 2-dimensional surface in $\mathbb{L}^{3}$ and $x: M \rightarrow \mathbb{L}^{3}$ a smooth nondegenerate isometric immersion. A surface $M$ is said to be space-like (resp. time-like) if the induced metric on $M$ is positive definite (resp. indefinite). Assuming that $M$ is orientable, we can always choose a unit normal vector field $G$ globally defined on $M$. In such a case, the unit normal vector field $G$ can be regarded as a map $G: M \rightarrow \mathbb{H}_{+}^{2}$ if $M$ is space-like and as a map $G: M \rightarrow \mathbb{S}_{1}^{2}$ if $M$ is time-like, where $\mathbb{H}_{+}^{2}=\left\{x \in \mathbb{L}^{3} \mid\langle x, x\rangle=-1, x_{2}>0\right\}$ is the hyperbolic space and $\mathbb{S}_{1}^{2}=\left\{x \in \mathbb{L}^{3} \mid\langle x, x\rangle=1\right\}$ is the de Sitter space. The map $G$ is also called the Gauss map of the surface $M$. For the matrix $\tilde{g}=\left(\tilde{g}_{i j}\right)$ consisting of the components of the induced metric on $M$, we denote by $\tilde{g}^{-1}=\left(\tilde{g}^{i j}\right)$ (resp. $\mathcal{G}$ ) the inverse matrix (resp. the determinant) of the matrix $\left(\tilde{g}_{i j}\right)$. The Laplacian $\Delta$ on $M$ is, in turn, given by

$$
\Delta=-\frac{1}{\sqrt{|\mathcal{G}|}} \sum_{i, j} \frac{\partial}{\partial x^{i}}\left(\sqrt{|\mathcal{G}|} \tilde{g}^{i j} \frac{\partial}{\partial x^{j}}\right) .
$$

Let $e$ be a non-zero vector in $\mathbb{L}^{3}$ and $\mathbf{S}(e)$ the set of screw motions fixing $e$ in $\mathbb{L}^{3}$. In particular, if $e$ is non-null, the screw motions fixing $e$ belong to 
$\mathbf{O}(e)$, the set of orthogonal transformations with positive determinant. Then, a helicoidal motion around the axis in the $e$-direction is defined by

$$
g_{t}(x)=A(t) x^{T}+(h t) e, x=\left(x_{0}, x_{1}, x_{2}\right) \in \mathbb{L}^{3}, t \in \mathbb{R}, A \in \mathbf{S}(e),
$$

where $h$ is a constant and $x^{T}$ is the transpose of the vector $x$.

Let $\gamma: I=(a, b) \subset \mathbb{R} \rightarrow \Pi$ be a plane curve in $\mathbb{L}^{3}$ and $l$ a straight line in $\Pi$ which does not intersect the curve $\gamma$. A helicoidal surface $M$ with the axis $l$ and pitch $h$ in $\mathbb{L}^{3}$ is a non-degenerate surface which is invariant under the action of the helicoidal motion $g_{t}$. Depending on the axis being space-like, time-like or null, there are three types of screw motions. If the axis $l$ is space-like (resp. time-like), then $l$ is transformed to the $x_{1}$-axis or $x_{2}$-axis (resp. $x_{0}$-axis) by the Lorentz transformation. Therefore, we may consider $x_{2}$-axis (resp. $x_{0}$-axis) as the axis if $l$ is space-like (resp. time-like). If the axis $l$ is null, then we may assume that the axis is the line spanned by the vector $(1,1,0)$.

We now consider the helicoidal surfaces in $\mathbb{L}^{3}$ with space-like, time-like or null axis respectively.

Case 1. The axis $l$ is space-like.

Without loss of generality we may assume that the profile curve $\gamma$ lies in the $x_{1} x_{2}$-plane or $x_{0} x_{2}$-plane. Hence, the curve $\gamma$ can be represented by

$$
\gamma(u)=(0, f(u), g(u)) \text { or } \gamma(u)=(f(u), 0, g(u))
$$

for smooth functions $f$ and $g$ on an open interval $I=(a, b)$. Therefore, the surface $M$ may be parameterized by

$$
x(u, v)=(f(u) \sinh v, f(u) \cosh v, g(u)+h v), f(u)>0, h \in \mathbb{R}
$$

or

$$
x(u, v)=(f(u) \cosh v, f(u) \sinh v, g(u)+h v), f(u)>0, h \in \mathbb{R} .
$$

Case 2. The axis $l$ is time-like.

In this case, we may assume that the profile curve $\gamma$ lies in the $x_{0} x_{1}$-plane. So the curve $\gamma$ is given by $\gamma(u)=(g(u), f(u), 0)$ for a positive function $f=f(u)$ on an open interval $I=(a, b)$. Hence, the surface $M$ can be expressed by

$$
x(u, v)=(g(u)+h v, f(u) \cos v, f(u) \sin v), f(u)>0, h \in \mathbb{R} .
$$

Case 3. The axis $l$ is null.

In this case, we may assume that the profile curve $\gamma$ lies in the $x_{0} x_{1}$-plane of the form $\gamma(u)=(f(u), g(u), 0)$, where $f=f(u)$ is a positive function and $g=g(u)$ is a function satisfying $p(u)=f(u)-g(u) \neq 0$ for all $u \in I$. Under the cubic screw motion, its parametrization has the form

$$
x(u, v)=\left(f(u)+\frac{v^{2}}{2} p(u)+h v, g(u)+\frac{v^{2}}{2} p(u)+h v, p(u) v\right), h \in \mathbb{R} .
$$




\section{Helicoidal surfaces with time-like axis in Minkowski 3-space}

In this section, we study the helicoidal surfaces with harmonic Gauss map which has time-like axis in Minkowski 3-space $\mathbb{L}^{3}$.

Suppose that $M$ is a helicoidal surface in $\mathbb{L}^{3}$ with time-like axis parameterized by (2.3) for some smooth functions $f$ and $g$.

First, if $f$ is constant, the parametrization of $M$ can be written as

$$
x(u, v)=(g(u)+h v, a \cos v, a \sin v), h \in \mathbb{R}
$$

for a non-zero constant $a$. By a straightforward computation, we see that the Laplacian $\Delta G$ of the Gauss map $G$ satisfies $\Delta G=\frac{1}{a^{2}} G$. Hence, $M$ does not have the harmonic Gauss map. In fact, it has non-proper pointwise 1-type Gauss map of the first kind ([5]). Therefore, we may assume that $f$ is not constant. Then, we may put $f(u)=u$ and thus $M$ is parameterized by

$$
x(u, v)=(g(u)+h v, u \cos v, u \sin v), u>0, h \in \mathbb{R} .
$$

If $M$ is space-like, that is, $u^{2}-u^{2} g^{\prime 2}-h^{2}>0$, then the Gauss map $G$ and its Laplacian $\Delta G$ are obtained as follows:

$$
G=\frac{1}{\sqrt{u^{2}-u^{2} g^{\prime 2}-h^{2}}}\left(-u,-u g^{\prime} \cos v+h \sin v,-u g^{\prime} \sin v-h \cos v\right)
$$

and

$\Delta G=-\frac{1}{\left(u^{2}-u^{2} g^{\prime 2}-h^{2}\right)^{\frac{7}{2}}}(D(u), A(u) \sin v+B(u) \cos v,-A(u) \cos v+B(u) \sin v)$, where we have put

$$
\begin{aligned}
A(u)= & h\left\{2 h^{4}-4 h^{4} g^{\prime 2}+\left(-7 h^{4} g^{\prime} g^{\prime \prime}\right) u+\left(-2 h^{2}+2 h^{2} g^{2}-h^{4} g^{\prime \prime 2}-h^{4} g^{\prime} g^{\prime \prime \prime}\right) u^{2}\right. \\
& +\left(8 h^{2} g^{\prime} g^{\prime \prime}+h^{2} g^{\prime 3} g^{\prime \prime}\right) u^{3}+\left(3 h^{2} g^{\prime 2} g^{\prime \prime 2}-h^{2} g^{3} g^{\prime \prime \prime}+2 h^{2} g^{\prime \prime 2}+2 h^{2} g^{\prime} g^{\prime \prime \prime}\right) u^{4} \\
& \left.+\left(-g^{\prime} g^{\prime \prime}+g^{\prime 3} g^{\prime \prime}\right) u^{5}+\left(-g^{\prime \prime 2}-3 g^{\prime 2} g^{\prime \prime 2}-g^{\prime} g^{\prime \prime \prime}+g^{\prime 3} g^{\prime \prime \prime}\right) u^{6}\right\} \\
B(u)= & -3 h^{6} g^{\prime \prime}+\left(-6 h^{4} g^{\prime}+8 h^{4} g^{\prime 3}-h^{6} g^{\prime \prime \prime}\right) u+\left(7 h^{4} g^{\prime \prime}+7 h^{4} g^{\prime 2} g^{\prime \prime}\right) u^{2} \\
& +\left(7 h^{2} g^{\prime}-12 h^{2} g^{\prime 3}+5 h^{2} g^{\prime 5}+4 h^{4} g^{\prime} g^{\prime \prime 2}+3 h^{4} g^{\prime \prime \prime}-h^{4} g^{\prime 2} g^{\prime \prime \prime}\right) u^{3} \\
& +\left(-5 h^{2} g^{\prime \prime}-6 h^{2} g^{\prime 2} g^{\prime \prime}+2 h^{2} g^{\prime} g^{\prime \prime}\right) u^{4}+\left\{-g^{\prime}\left(1-g^{\prime 2}\right)^{3}-8 h^{2} g^{\prime} g^{\prime \prime 2}\right. \\
& \left.-3 h^{2} g^{\prime \prime \prime}+2 h^{2} g^{\prime 2} g^{\prime \prime \prime}\right\} u^{5}+\left(g^{\prime \prime}-g^{\prime 2} g^{\prime \prime}\right) u^{6}+\left(-g^{\prime 2} g^{\prime \prime \prime}+4 g^{\prime} g^{\prime \prime 2}+g^{\prime \prime \prime}\right) u^{7}
\end{aligned}
$$

and

$$
\begin{aligned}
D(u)= & u\left\{-2 h^{4}+4 h^{4} g^{\prime 2}+\left(7 h^{4} g^{\prime} g^{\prime \prime}\right) u+\left(2 h^{2}-2 h^{2} g^{2}+h^{4} g^{\prime \prime 2}+h^{4} g^{\prime} g^{\prime \prime \prime}\right) u^{2}\right. \\
& +\left(-8 h^{2} g^{\prime} g^{\prime \prime}-h^{2} g^{\prime 3} g^{\prime \prime}\right) u^{3}+\left(-3 h^{2} g^{\prime 2} g^{\prime \prime 2}+h^{2} g^{\prime 3} g^{\prime \prime \prime}-2 h^{2} g^{\prime \prime 2}\right. \\
& \left.\left.-2 h^{2} g^{\prime} g^{\prime \prime \prime}\right) u^{4}+\left(g^{\prime} g^{\prime \prime}-g^{\prime} g^{\prime \prime}\right) u^{5}+\left(g^{\prime \prime 2}+3 g^{\prime 2} g^{\prime \prime 2}+g^{\prime} g^{\prime \prime \prime}-g^{\prime 3} g^{\prime \prime \prime}\right) u^{6}\right\} .
\end{aligned}
$$

Suppose that $M$ has harmonic Gauss map, that is, its Gauss map $G$ satisfies $\Delta G=0$. Then, we obtain that the functions $A(u), B(u)$ and $D(u)$ are all vanishing. 
First, we consider the case that $M$ is a helicoidal surface of polynomial kind with harmonic Gauss map, that is, $g$ is a polynomial in $\mathrm{u}$. Then we may put

$$
g(u)=a_{n} u^{n}+a_{n-1} u^{n-1}+\cdots+a_{1} u+a_{0},
$$

where $n$ is nonnegative integer and $a_{n}$ is non-zero constant.

Considering the constant terms of $B(u)$, it is easy to see that $h=0$, that is, $M$ is a surface of revolution. Therefore, $A(u)=0$. Also, $B(u)$ and $D(u)$ are reduced to respectively:

$$
\begin{aligned}
& B(u)=-g^{\prime}\left(1-g^{\prime 2}\right)^{3} u^{5}+\left(g^{\prime \prime}-g^{\prime 2} g^{\prime \prime}\right) u^{6}+\left(-g^{\prime 2} g^{\prime \prime \prime}+4 g^{\prime} g^{\prime \prime 2}+g^{\prime \prime \prime}\right) u^{7}, \\
& D(u)=\left(g^{\prime} g^{\prime \prime}-g^{\prime 3} g^{\prime \prime}\right) u^{5}+\left(g^{\prime \prime 2}+3 g^{\prime 2} g^{\prime \prime 2}+g^{\prime} g^{\prime \prime \prime}-g^{\prime 3} g^{\prime \prime \prime}\right) u^{6} .
\end{aligned}
$$

Assume that $\operatorname{deg} g(u) \geq 2$, where $\operatorname{deg} g(u)$ means the degree of the polynomial $g(u)$. Then, the term $-g^{\prime}\left(1-g^{\prime 2}\right)^{3} u^{5}$ in $B(u)$ includes the highest degree in $u$ and its leading coefficient must be zero, that is, $n^{7} a_{n}^{7}=0$. Thus, $a_{n}=0$, a contradiction.

Assuming $\operatorname{deg} g(u)=1, B(u)=-a_{1}\left(1-a_{1}^{2}\right)^{3} u^{5}$. Hence, $a_{1}^{2}=1$, which is a contradiction since $M$ is non-degenerate.

If $g$ is constant, then $B(u)=0$ and $D(u)=0$. Hence, the Gauss map is harmonic. In this case, the parametrization of $M$ in (3.1) is reduced to

$$
x(u, v)=(a, u \cos v, u \sin v), u>0
$$

for some constant $a$. This means that $M$ is part of a plane.

Conversely, it is obvious that the Gauss map of a plane is harmonic. By a similar process as above, the same conclusion can be made in case of time-like surface. Consequently, we have:

Theorem 3.1. Let $M$ be a helicoidal surface of polynomial kind with time-like axis in a Minkowski 3-space $\mathbb{L}^{3}$. Then, $M$ has the harmonic Gauss map if and only if $M$ is part of a plane.

Next, consider $M$ is of rational kind, that is, $g(u)$ is a rational function. Suppose that $M$ is a genuine helicoidal surface of rational kind with harmonic Gauss map, i.e., $h \neq 0$. Then we may put

$$
g(u)=p(u)+\frac{r(u)}{q(u)},
$$

where $p(u)$ is a polynomial in $u$ and the polynomials $r(u)$ and $q(u)$ are relatively prime with $\operatorname{deg} r(u)<\operatorname{deg} q(u)$ and $\operatorname{deg} q(u) \geq 1$. Let $\operatorname{deg} p(u)=l, \operatorname{deg} r(u)=$ $n$ and $\operatorname{deg} q(u)=m$ with $n<m$ and $m \geq 1$ where $l, m$ and $n$ are some nonnegative integers. Then, we may put

$$
\begin{aligned}
& p(u)=a_{l} u^{l}+a_{l-1} u^{l-1}+\cdots+a_{1} u+a_{0}, \\
& q(u)=b_{m} u^{m}+b_{m-1} u^{m-1}+\cdots+b_{1} u+b_{0}, \\
& r(u)=c_{n} u^{n}+c_{n-1} u^{n-1}+\cdots+c_{1} u+c_{0} .
\end{aligned}
$$


Putting (3.2) in the equation $B(u)$ and multiplying $q^{14}(u)$ with thus obtained equation, we get a polynomial $q^{14}(u) B(u)$ in $u$.

Assume that $\operatorname{deg} p(u) \geq 2$. By an algebraic computation, we see that the degree of the polynomial is $7 l+14 m-2$ and so its coefficient $l^{7} a_{l}^{7} b_{m}^{14}$ must be zero. But, this is a contradiction.

Assuming $\operatorname{deg} p(u)=1$, the leading coefficient of the polynomial is $-a_{1}(1-$ $\left.a_{1}^{2}\right)^{3} b_{m}^{14}$. It must be zero and so $a_{1}^{2}=1$. In this case, we can consider two cases according to the value of $m-n$.

If $m-n>1$, then the polynomial includes the term of the degree $14 m+1$ with the coefficient $2 h^{4} a_{1} b_{m}^{14}$. Hence it must be zero, a contradiction.

Suppose $m-n=1$. Since the Gauss map of $M$ is harmonic, the polynomials $q^{10}(u) A(u)$ and $q^{14}(u) B(u)$ are vanishing. With the help of (3.2) and (3.3), we have $b_{0}=0$. So we may put

$$
q(u)=b_{m} u^{m}+\cdots+b_{2} u^{2}+b_{1} u, b_{m} \neq 0 .
$$

Then, an algebraic computation shows that the polynomial $q^{10}(u) A(u)$ has the lowest degree 4 with the coefficient $4 h^{2} b_{1}^{6} c_{0}^{4}$. Similarly, the polynomial $q^{14}(u) B(u)$ has the lowest degree 5 with the coefficient $-b_{1}^{7} c_{0}^{7}$. Therefore, $b_{1} c_{0}=$ 0 .

If we assume $c_{0} \neq 0$, then $b_{1}=0$ and we have

$$
q(u)=b_{m} u^{m}+\cdots+b_{2} u^{2}, b_{m} \neq 0 .
$$

By considering the coefficients of the terms with the lowest degree in $q^{10}(u) A(u)$ and $q^{14}(u) B(u)$, we get $b_{2} c_{0}=0$. Hence, $b_{2}=0$. Inductively, $b_{3}, \ldots, b_{m-1}$ are zero. So we put

$$
q(u)=b_{m} u^{m}, b_{m} \neq 0 .
$$

Then, the polynomial $q^{14}(u) B(u)$ has the lowest degree $7 m-2$ with the coefficient $\left(-m b_{m} c_{0}\right)^{7}$. It must be zero, a contradiction. Thus, we conclude that $c_{0}=0$. Hence, $g(u)$ can be written as

$$
g(u)= \pm u+a_{0}+\frac{r(u)}{q(u)}
$$

where $r(u)=c_{n} u^{n-1}+\cdots+c_{1}$ and $q(u)=b_{m} u^{m-1}+\cdots+b_{1}$ with $c_{n} \neq 0$ and $b_{m} \neq 0$. By a similar process as above, we obtain $b_{1}, \ldots, b_{m-1}=0$ and $c_{1}, \ldots, c_{n-1}=0$. Consequently, we get

$$
g(u)= \pm u+a_{0}+\frac{c}{u}, c \neq 0 .
$$

Hence, $q^{14}(u) B(u)$ has the coefficient $-c^{7}$ of the lowest degree which is 5 and it must be zero. Thus, $c=0$, that is, $g$ is a polynomial in $u$.

Finally, if $p$ is constant, then the degree of $q^{14}(u) B(u)$ is $13 m+n+4$ and its leading coefficient is $-(m-n)^{2}(m-n+2) b_{m}^{13} c_{n}$. This must be zero, a contradiction. 
By a similar argument as above, we lead to a contradiction in case of surfaces of revolution. In case of time-like surface, we have the same result. Consequently, we have:

Theorem 3.2. Let $M$ be a helicoidal surface with time-like axis in a Minkowski 3-space $\mathbb{L}^{3}$. Then, there exists no helicoidal surface of rational kind with harmonic Gauss map except polynomial kind.

Combining the above theorems we have the following:

Theorem 3.3 (Characterization). Let $M$ be a rational helicoidal surface with time-like axis in a Minkowski 3-space $\mathbb{L}^{3}$. Then, $M$ has the harmonic Gauss map if and only if it is part of a plane.

Combining the results above and [5], we have the following characterization.

Theorem 3.4 (Characterization). Let $M$ be a rational helicoidal surface with time-like axis in a Minkowski 3-space $\mathbb{L}^{3}$. Then, the Gauss map $G$ of $M$ satisfies the condition $\Delta G=F(G+C)$ for some smooth function $F$ and constant vector $C$ if and only if $M$ is an open part of a plane, a circular cylinder, a right cone, a right helicoid of type II or a helicoidal surface of elliptic type in $\mathbb{L}^{3}$.

\section{Helicoidal surfaces with null axis in Minkowski 3-space}

In this section, we investigate the helicoidal surfaces with harmonic Gauss map which has null axis in $\mathbb{L}^{3}$.

Suppose that $M$ is a helicoidal surface with null axis parameterized by

$$
x(u, v)=\left(f(u)+\frac{v^{2}}{2} p(u)+h v, g(u)+\frac{v^{2}}{2} p(u)+h v, p(u) v\right), h \in \mathbb{R},
$$

where $p(u)=f(u)-g(u) \neq 0$. Since the induced metric on $M$ is non-degenerate, $(f(u)-g(u))^{2}\left(f^{\prime 2}(u)-g^{\prime 2}(u)\right)+h^{2}\left(f^{\prime}(u)-g^{\prime}(u)\right)^{2}$ never vanishes and so $f^{\prime}(u)-$ $g^{\prime}(u) \neq 0$ everywhere. Thus, we may change the variable in such a way that $p(u)=f(u)-g(u)=-2 u$.

Let $k(u)=f(u)+u$. Then, the functions $f$ and $g$ in the profile curve $\gamma$ look like

$$
f(u)=k(u)-u \text { and } g(u)=k(u)+u .
$$

Thus, the parametrization of $M$ becomes

$$
x(u, v)=\left(k(u)-u-u v^{2}+h v, k(u)+u-u v^{2}+h v,-2 u v\right) .
$$

We now suppose that $M$ is space-like, that is, $4 u^{2} k^{\prime}(u)-h^{2}>0$. By a direct computation, the Gauss map $G$ and its Laplacian $\Delta G$ are obtained as follows: $G=\frac{1}{\sqrt{4 u^{2} k^{\prime}(u)-h^{2}}}\left(u k^{\prime}(u)+u+u v^{2}-v h, u k^{\prime}(u)-u+u v^{2}-v h, 2 u v-h\right)$ and

$$
\Delta G=-\frac{1}{\left(4 u^{2} k^{\prime}(u)-h^{2}\right)^{\frac{7}{2}}}(2 u X+Y,-2 u X+Y, 2(2 u v-h) X),
$$


where we have put

(4.1)

$X=X(u)=h^{4}+4 h^{2} k^{\prime} u^{2}+9 h^{2} k^{\prime \prime} u^{3}+h^{2} k^{\prime \prime \prime} u^{4}-4 k^{\prime} k^{\prime \prime} u^{5}+8 k^{\prime \prime 2} u^{6}-4 k^{\prime} k^{\prime \prime \prime} u^{6}$

and

$$
\begin{aligned}
Y= & Y(u, v) \\
= & 10 h^{4} k^{\prime} u+7 h^{4} k^{\prime \prime} u^{2}-32 h^{2}{k^{\prime}}^{2} u^{3}+h^{4} k^{\prime \prime \prime} u^{3}-14 h^{2} k^{\prime} k^{\prime \prime} u^{4}+32 k^{3} u^{5} \\
& +6 h^{2} k^{\prime \prime 2} u^{5}-6 h^{2} k^{\prime} k^{\prime \prime \prime} u^{5}+8 k^{\prime 2} k^{\prime \prime} u^{6}-8 k^{\prime} k^{\prime \prime 2} u^{7}+8 k^{\prime 2} k^{\prime \prime \prime} u^{7}-2 h^{5} v \\
& -8 h^{3} k^{\prime} u^{2} v-18 h^{3} k^{\prime \prime} u^{3} v-2 h^{3} k^{\prime \prime \prime} u^{4} v+8 h k^{\prime} k^{\prime \prime} u^{5} v-16 h k^{\prime \prime 2} u^{6} v \\
& +8 h k^{\prime} k^{\prime \prime \prime} u^{6} v+2 h^{4} u v^{2}+8 h^{2} k^{\prime} u^{3} v^{2}+18 h^{2} k^{\prime \prime} u^{4} v^{2}+2 h^{2} k^{\prime \prime \prime} u^{5} v^{2} \\
& -8 k^{\prime} k^{\prime \prime} u^{6} v^{2}+16 k^{\prime \prime} u^{7} v^{2}-8 k^{\prime} k^{\prime \prime \prime} u^{7} v^{2} .
\end{aligned}
$$

Suppose that $M$ has harmonic Gauss map, that is, its Gauss map $G$ satisfies $\Delta G=0$. Then the above equations $X(u)$ and $Y(u, v)$ are vanishing. Hence, the equation $Y(u, v)$ in (4.2) can be rewritten as

$$
Y(u, v)=Y_{1}(u)+Y_{2}(u) v+Y_{3}(u) v^{2},
$$

where we put

$$
\begin{gathered}
Y_{1}(u)=10 h^{4} k^{\prime} u+7 h^{4} k^{\prime \prime} u^{2}-32 h^{2} k^{2} u^{3}+h^{4} k^{\prime \prime \prime} u^{3}-14 h^{2} k^{\prime} k^{\prime \prime} u^{4}+32 k^{3} u^{5} \\
+6 h^{2} k^{\prime \prime 2} u^{5}-6 h^{2} k^{\prime} k^{\prime \prime \prime} u^{5}+8 k^{\prime 2} k^{\prime \prime} u^{6}-8 k^{\prime} k^{\prime \prime 2} u^{7}+8 k^{\prime 2} k^{\prime \prime \prime} u^{7}, \\
Y_{2}(u)=-2 h\left(h^{4}+4 h^{2} k^{\prime} u^{2}+9 h^{2} k^{\prime \prime} u^{3}+h^{2} k^{\prime \prime \prime} u^{4}-4 k^{\prime} k^{\prime \prime} u^{5}+8 k^{\prime \prime 2} u^{6}-4 k^{\prime} k^{\prime \prime \prime} u^{6}\right), \\
Y_{3}(u)=2 u\left(h^{4}+4 h^{2} k^{\prime} u^{2}+9 h^{2} k^{\prime \prime} u^{3}+h^{2} k^{\prime \prime \prime} u^{4}-4 k^{\prime} k^{\prime \prime} u^{5}+8 k^{\prime \prime 2} u^{6}-4 k^{\prime} k^{\prime \prime \prime} u^{6}\right) .
\end{gathered}
$$

Since $X(u)$ and $Y(u, v)$ are vanishing, we have $Y_{1}(u)=0$. Moreover, $Y_{1}(u)$ can be written as $Y_{1}(u)=-2 k^{\prime} u X(u)+u Z(u)$ and we also get $Z(u)=0$, where

$$
\begin{aligned}
& Z(u)=12 h^{4} k^{\prime}+7 h^{4} k^{\prime \prime} u-24 h^{2}{k^{\prime}}^{2} u^{2}+h^{4} k^{\prime \prime \prime} u^{2}+4 h^{2} k^{\prime} k^{\prime \prime} u^{3}+32 k^{\prime 3} u^{4} \\
& +6 h^{2}{k^{\prime \prime}}^{2} u^{4}-4 h^{2} k^{\prime} k^{\prime \prime \prime} u^{4}+8 k^{\prime} k^{\prime \prime 2} u^{6} \text {. }
\end{aligned}
$$

Let $M$ be a helicoidal surface of polynomial kind with harmonic Gauss map, that is, $k$ is a polynomial in $u$. Then we may put

$$
k(u)=a_{n} u^{n}+a_{n-1} u^{n-1}+\cdots+a_{1} u+a_{0},
$$

where $n$ is nonnegative integer and $a_{n}$ is non-zero constant.

Considering the constant terms in $X(u)$, it is easy to see that $h=0$. Therefore, the equations $X(u)$ and $Z(u)$ can be written as

$$
X(u)=-4 k^{\prime} k^{\prime \prime} u^{5}+8 k^{\prime \prime 2} u^{6}-4 k^{\prime} k^{\prime \prime \prime} u^{6} \text { and } Z(u)=32{k^{\prime}}^{3} u^{4}+8 k^{\prime} k^{\prime \prime 2} u^{6} .
$$

Assume that $\operatorname{deg} k(u) \geq 2$. Considering the equation $X(u)$, we can easily lead to a contradiction. 
If $\operatorname{deg} k(u)=1$, then $X(u)=0$ and $Z(u)=32 a_{1}^{3} u^{4}$. Hence, $Z(u)$ cannot be zero and so we have a contradiction.

If $k$ is constant, then $X(u)=0$ and $Z(u)=0$. But, in this case, it contradicts that $M$ is non-degenerate, i.e., $4 u^{2} k^{\prime}(u) \neq 0$. Hence, $M$ does not have harmonic Gauss map.

By a similar argument as above, we have the same results in case of time-like helicoidal surface of polynomial kind with null axis. Thus, we have:

Theorem 4.1. Suppose that $M$ is a helicoidal surface of polynomial kind with null axis in a Minkowski 3-space $\mathbb{L}^{3}$. Then $M$ does not have harmonic Gauss map.

We now consider a helicoidal surface of rational kind with harmonic Gauss map, that is, $k$ is a rational function in $u$. Then we may put

$$
k(u)=p(u)+\frac{r(u)}{q(u)},
$$

where $p(u)$ is a polynomial in $u, r(u)$ and $q(u)$ are relatively prime polynomials with $\operatorname{deg} r(u)<\operatorname{deg} q(u)$ and $\operatorname{deg} q(u) \geq 1$.

Suppose that $M$ is a genuine helicoidal surface of rational kind, that is, $h \neq 0$. With the help of (4.1) and (4.3), we get

$u^{2} Z(u)-h^{2} X(u)=\left(4 u^{2} k^{\prime}-h^{2}\right)\left(h^{4}-4 h^{2} k^{\prime} u^{2}+2 h^{2} k^{\prime \prime} u^{3}+8 k^{\prime 2} u^{4}+2 k^{\prime \prime 2} u^{6}\right)$.

Since $X(u)$ and $Z(u)$ vanishes identically,

$$
\left(4 u^{2} k^{\prime}-h^{2}\right)\left(h^{4}-4 h^{2} k^{\prime} u^{2}+2 h^{2} k^{\prime \prime} u^{3}+8{k^{\prime}}^{2} u^{4}+2{k^{\prime \prime}}^{2} u^{6}\right)=0 .
$$

Because $M$ is a nondegenerate surface, i.e., $4 u^{2} k^{\prime}-h^{2} \neq 0$,

$$
h^{4}-4 h^{2} k^{\prime} u^{2}+2 h^{2} k^{\prime \prime} u^{3}+8{k^{\prime}}^{2} u^{4}+2{k^{\prime \prime}}^{2} u^{6}=0 .
$$

From the equation (4.4), we get

$$
\left(2 k^{\prime \prime} u^{3}+h^{2}\right)^{2}+\left(4 u^{2} k^{\prime}-h^{2}\right)^{2}=0 .
$$

It is easily seen that this is a contradiction because of $4 u^{2} k^{\prime}-h^{2} \neq 0$. Thus, $h=0$.

If $h=0$, the equation $Z(u)$ in $(4.3)$ can be reduced as

$$
Z(u)=8 u^{2} k^{\prime}\left(k^{\prime \prime 2} u^{4}+4 u^{2}{k^{\prime}}^{2}\right) .
$$

Since $M$ is nondegenerate, $k^{\prime \prime 2} u^{4}+4 u^{2}{k^{\prime}}^{2}=0$, which implies $k$ is constant, a contradiction.

Similarly, we prove that a time-like helicoidal surface of rational kind does not have harmonic Gauss map. Consequently, we have:

Theorem 4.2. Let $M$ be a helicoidal surface with null axis in a Minkowski 3-space $\mathbb{L}^{3}$. Then, there exists no rational helicoidal surface with harmonic Gauss map. 
Combining the results we obtained above and those in [5], we have the following:

Theorem 4.3 (Characterization). Let $M$ be a helicoidal surface of rational kind with null axis in a Minkowski 3-space $\mathbb{L}^{3}$. Then, the Gauss map $G$ of $M$ satisfies $\Delta G=F(G+C)$ for some smooth function $F$ and constant vector $C$ if and only if it is part of an Enneper's surface of second kind, a de Sitter space, a hyperbolic space, a helicoidal surface of Enneper type, a helicoidal surface of hyperbolic type or a helicoidal surface of de Sitter type in $\mathbb{L}^{3}$.

\section{References}

[1] C. Baikoussis and L. Verstraelen, On the Gauss map of helicoidal surfaces, Rend. Sem. Mat. Messina Ser. II 2(16) (1993), 31-42.

[2] C. C. Beneki, G. Kaimakamis, and B. J. Papantoniou, Helicoidal surfaces in threedimensional Minkowski space, J. Math. Anal. Appl. 275 (2002), no. 2, 586-614.

[3] B.-Y. Chen, Total Mean Curvature and Submanifolds of Finite Type, Series in Pure Mathematics, 1. World Scientific Publishing Co., Singapore, 1984.

[4] M. Choi, D. S. Kim, and Y. H. Kim, Helicoidal surfaces with pointwise 1-type Gauss map, to appear in J. Korean Math. Soc.

[5] M. Choi, Y. H. Kim, H. Liu, and D. W. Yoon, Helicoidal surfaces and their Gauss map in Minkowski 3-space, submitted for publication.

[6] F. Ji and Z. H. Hou, Helicoidal surfaces under the cubic screw motion in Minkowski 3-space, J. Math. Anal. Appl. 318 (2006), no. 2, 634-647.

[7] Y. H. Kim and D. W. Yoon, Classification of ruled surfaces in Minkowski 3-spaces, J. Geom. Phys. 49 (2004), no. 1, 89-100.

[8] - On the Gauss map of ruled surfaces in Minkowski space, Rocky Mountain J. Math. 35 (2005), no. 5, 1555-1581.

Miekyung Choi

Department of Mathematics

KyungPook National University

TAEGU 702-701, KOREA

E-mail address: mkchoi@knu.ac.kr

Young Ho KIM

Department of Mathematics

KyUNGPOOK NATIONAL UNIVERSity

TAEgu 702-701, Korea

E-mail address: yhkim@knu.ac.kr

Gi-Chan PARK

Department of Mathematics

KyungPoOK National University

TAEGU 702-701, Korea 